\title{
Alterations Of Glycerophospholipid And Fatty Acyl Metabolism In Multiple Brain Regions Of Schizophrenia Microbiota Recipient Mice
}

This article was published in the following Dove Press journal: Neuropsychiatric Disease and Treatment

\author{
Weiwei Liang ${ }^{1-3, *}$ \\ Yu Huang $1,3, *$ \\ Xunmin $\operatorname{Tan}^{1,3, *}$ \\ Jing $\mathrm{Wu} \mathbb{1}^{3,4}$ \\ Jiajia Duan ${ }^{3,4}$ \\ Hanping Zhang ${ }^{1,3}$ \\ Bangmin Yin ${ }^{1,3}$ \\ Yifan $\mathrm{Li}^{1,3}$ \\ Peng Zheng ${ }^{1,3}$ \\ Hong Wei ${ }^{5}$ \\ Peng $\mathrm{Xie}^{1,3}$
}

'Department of Neurology, The First Affiliated Hospital of Chongqing Medical University, Chongqing 400016, People's Republic of China; ${ }^{2}$ Department of Neurology, Yongchuan Hospital, Chongqing Medical University, Chongqing 402460, People's Republic of China; ${ }^{3}$ Institute of Neuroscience and the Collaborative Innovation Center for Brain Science, Chongqing Medical University, Chongqing 400016, People's Republic of China; ${ }^{4}$ The M.O.E. Key Laboratory of Laboratory Medical Diagnostics, The College of Laboratory Medicine, Chongqing Medical University, Chongqing 400016, People's Republic of China; ${ }^{5}$ Precision Medicine Institute, The First Affiliated Hospital, Sun Yat-Sen University, Guangzhou, Guangdong 510080, People's Republic of China

*These authors contributed equally to this work

Correspondence: Peng Xie

Department of Neurology, The First

Affiliated Hospital of Chongqing Medical

University, I Youyi Road, Yuzhong

District, Chongqing 4000 I6, People's

Republic of China

Tel +86-23-68485490

$\mathrm{Fax}+86-23-684851 \mathrm{II}$

Email xiepeng@cqmu.edu.cn
Background: Schizophrenia is a debilitating psychiatric disorder characterized by molecular and anatomical abnormalities of multiple brain regions. Our recent study showed that dysbiosis of the gut microbiota contributes to the onset of schizophrenia-relevant behaviors, but the underlying mechanisms remain largely unknown.

Purpose: This study aimed to investigate how gut microbiota shapes metabolic signatures in multiple brain regions of schizophrenia microbiota recipient mice.

Methods: Gas chromatography-mass spectrometry (GC-MS) and liquid chromatographymass spectrometry (LC-MS) were used to compare the metabolic signatures in the cortex, cerebellum and striatum of schizophrenia microbiota and healthy microbiota recipient mice. Enrichment analysis was further conducted to uncover the crucial metabolic pathways related to schizophrenia-relevant behaviors.

Results: We found that the metabolic phenotypes of these three regions were substantially different in schizophrenia microbiota recipient mice from those in healthy microbiota recipient mice. In total, we identified 499 differential metabolites that could discriminate the two groups in the three brain regions. These differential metabolites were mainly involved in glycerophospholipid and fatty acyl metabolism. Moreover, we found four of fatty acyl metabolites that were consistently altered in the three brain regions.

Conclusion: Taken together, our study suggests that alterations of glycerophospholipid and fatty acyl metabolism are implicated in the onset of schizophrenia-relevant behaviors, which may provide a new understanding of the etiology of schizophrenia.

Keywords: schizophrenia, gut microbiota, metabolomics, glycerophospholipids, fatty acyls, fecal microbiota transplantation

\section{Introduction}

Schizophrenia is a chronic neurodevelopmental disorder characterized by abnormal mental activities such as impaired cognition, emotion and intention. ${ }^{1}$ It places a large economic burden on health systems, families and society. ${ }^{2}$ Increasing lines of evidence have suggested that social, environmental and genetic factors are implicated in the onset of this disease. ${ }^{3,4}$ Several classical theories, such as neurotransmitter alterations, gene loci and epigenetic abnormalities and dysregulation of inflammation, have attempted to explain the pathogenesis of schizophrenia. ${ }^{5-8}$ However, its underlying mechanisms remain largely unknown.

The gut microbiota is a large ecosystem that inhabits in the human intestine. ${ }^{9,10}$ Many studies have shown that the gut microbiota can modulate brain functions and 
behaviors through the microbiota-gut-brain axis. ${ }^{11-14}$ Clinical research has reported that schizophrenia is correlated with anatomical abnormalities of the gastrointestinal system. ${ }^{15}$ Moreover, our previous study showed that the gut microbial composition was significantly altered in schizophrenia patients relative to healthy controls, and dysbiosis of the gut microbiota contributed to the onset of schizophrenia-relevant behaviors through moudlation of the hippocampal glutamate-glutamine-GABA cycle. ${ }^{16}$ However, how the gut microbiota systematically shapes brain metabolic signatures remains unclear.

Abnormalities of neural circuits have been widely recognized as a hallmark of schizophrenia. ${ }^{17-19}$ Previous research demonstrated that brain regions such as the hippocampus, cortex and striatum are involved in the pathophysiology of schizophrenia. ${ }^{20,21}$ Additionally, clinical investigations have also shown that the cerebellum is associated with imparied cognition and emotion in schizophrenia patients. ${ }^{2-24}$ In this study, two metabolomics approaches, gas chromatography-mass spectrometry (GCMS) and liquid chromatography-mass spectrometry (LCMS), were combined to analyze the metabolic changes of three brain regions (cortex, cerebellum and striatumin) of schizophrenia microbiota and healthy microbiota recipient mice. Moreover, we mapped these differential metabolites to their respective biochemical pathways and further performed intersection metabolic analysis to determine how dysbiosis of the gut microbiota paticipates in the onset of schizophrenia.

\section{Materials And Methods}

\section{Animals And Sample Collection}

This animal study was approved by the Ethics Committee of Army Medical University (China) and Chongqing
Medical University (2011002). All experiments were performed according to the National Institutes of Health Guide for the Care and Use of Laboratory Animals. The details of our workflow are shown in Figure 1. The samples were derived from our previous research. ${ }^{16}$ The model was constructed by orally transplanting a liquid fecal suspension into germ-free mice. Briefly, the fecal microbiota transplantation (FMT) experiment was conducted by obtaining $0.2 \mathrm{~g}$ of feces from schizophrenia patients $(n=5)$ or healthy individuals $(n=5)$. These fecal samples were mixed with $3 \mathrm{~mL}$ of reduced sterile phosphate-buffered saline to obtain a suspension. Then, each mouse was randomly administered $200 \mu \mathrm{l}$ of the fecal suspension by gavage in a flexible-film gnotobiotic isolator. Behavioral tests including open-field, sociability and social novelty preference, prepulse inhibition, Y-maze and forced swimming tests were performed after 14 days of colonization. Consequently, we found that the germ-free mice that received schizophrenia microbiota transplants displayed schizophrenia-relevant behaviors relative to healthy microbiota recipient mice. All mice were sacrificed after the behavioral tests, and cortex, cerebellum and striatum tissues were collected and stored at $-80^{\circ} \mathrm{C}$ until metabolite detection.

\section{Gas Chromatography-Mass Spectrometry}

GC-MS metabolomics analysis was used to characterize the metabolic signatures of cortex, cerebellum and striatum tissues obtained from schizophrenia microbiota $(n=8)$ and healthy microbiota recipient mice $(n=8)$. Briefly, tissue samples $(30 \mathrm{mg})$ were placed in an Eppendorf tube $(1.5 \mathrm{~mL})$ with $20 \mu \mathrm{l}$ of an internal standard $(0.006 \mathrm{mg}$ of L-2-chloro-phenylalanine and $20 \mu \mathrm{l}$ of methanol) and $600 \mu \mathrm{l}$ of a mixture containing $480 \mu \mathrm{l}$ of methanol and $120 \mu \mathrm{l}$ of water. The

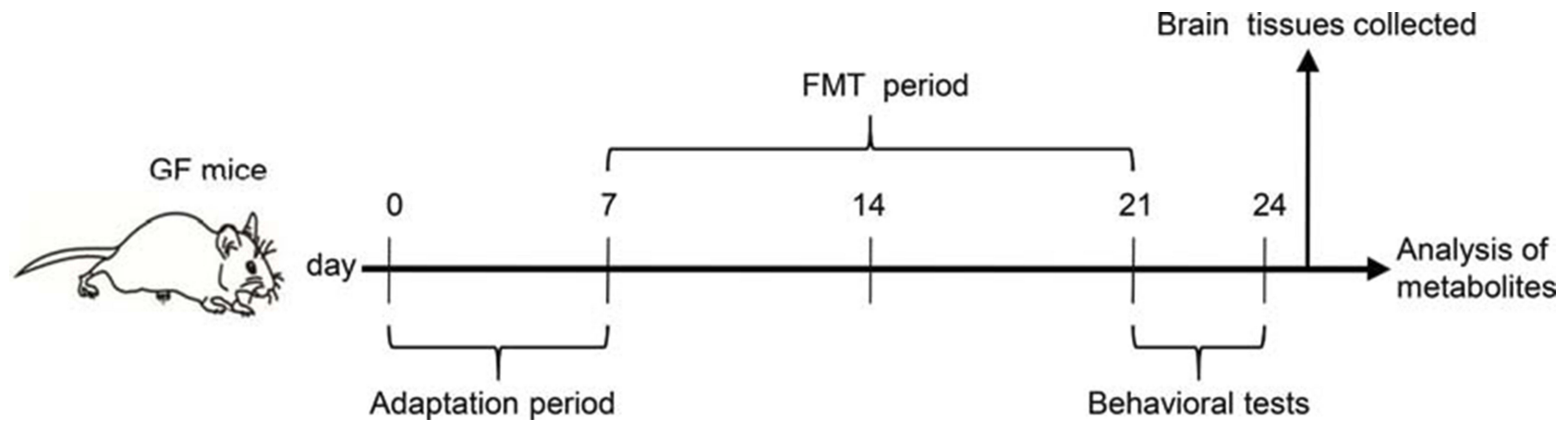

Figure I Experimental procedure. Day 0-7, animal adaptation time; animals were grouped on the last day of the first week; Day 8-2I, FMT period; Day 22-24, behavioral tests. Abbreviations: GF mice, germ-free mice; FMT, fecal microbiota transplantation. 
samples were extracted by ultrasonication for $600 \mathrm{~s}$ after homogenization and centrifuged at $4^{\circ} \mathrm{C}$ and $10,000 \mathrm{~g}$ for $900 \mathrm{~s}$ to obtain the dried extracts used for derivatization. Quality control (QC) samples were obtained by mixing all tissue samples and were analyzed using the same method used for the other samples. Every eighth QC sample was injected to assess the repeatability of the experiment. The tissue samples were analyzed by a gas chromatography system coupled to an MSD system (Agilent, CA). The derivative samples were separated using an HP-5MS capillary column made of fused silica. Highly purified helium with a steady speed of $6.0 \mathrm{~mL} / \mathrm{min}$ was used as the carrier gas. The temperature of the injection port was kept at $280^{\circ} \mathrm{C}$. ChromaTOF software was used to analyze the GC-MS data. Then, the Fiehn database was used to quantify the metabolites. Partial least-squares discriminant analysis (PLS-DA) was performed to visually discriminate the samples from schizophrenia microbiota and healthy microbiota recipient mice using SIMCA software (version 14.0, Umetrics, Umea, Sweden). ${ }^{25}$

\section{Liquid Chromatography-Mass Spectrometry}

Samples $(15 \mathrm{mg})$ were placed in an Eppendorf tube (1.5 $\mathrm{mL})$ with $20 \mu \mathrm{l}$ of internal standard $(0.006 \mathrm{mg}$ of L-2chloro-phenylalanine and $20 \mu \mathrm{l}$ of methanol) and $600 \mu \mathrm{l}$ of a mixture containing $480 \mu \mathrm{l}$ of methanol and $120 \mu \mathrm{l}$ of water. The samples were placed at $-80^{\circ} \mathrm{C}$ for $600 \mathrm{~s}$ and then centrifuged at $4^{\circ} \mathrm{C}$ and $10,000 \mathrm{~g}$ for $900 \mathrm{~s}$ after homogenization. Then, $150 \mu \mathrm{l}$ of supernatant fluid was used for the subsequent LC-MS analysis. QC samples were obtained by mixing all tissue samples and were analyzed using the same approach used for the other samples. Every eighth QC sample was injected to assess the repeatability of the experiment. The mobile phase consisted of aqueous formic acid $(0.1 \%(\mathrm{v} / \mathrm{v})$ formic acid) and acetonitrile $(0.1 \%(\mathrm{v} / \mathrm{v})$ formic acid); the injection volume was $3.00 \mu \mathrm{l}$, and the flow rate was set at 0.40 $\mathrm{mL} / \mathrm{min}$. A Waters mass spectrometer (VION IMS Q-TOF) was used to collect mass spectrometric data. The source and desolvation temperatures were set to $120^{\circ} \mathrm{C}$ and $500^{\circ}$ $\mathrm{C}$, respectively. The desolvation gas flow was set at $900 \mathrm{l} /$ $\mathrm{h}$. The range of centroid data was $50-1000 \mathrm{~m} / \mathrm{z}$. The scan time was set to $0.1 \mathrm{~s}$, and the inter scan time was set to $0.02 \mathrm{~s}$. The data were analyzed using Progenesis QI software before visualization. The SIMCA-P+14.0 software package was used to analyze the data set, which consisted of positive and negative data. PLS-DA was used to visualize the metabolic differences between the two groups. ${ }^{26}$ In this study, a seven-round cross-validation was performed to avoid model overfitting. The orthogonal projections to latent structures discriminant analysis models were also validated by a 200 -iteration permutation analysis. Detailed LC-MS analysis was performed according to our previously published study. ${ }^{13}$

\section{Metabolic Pathway Analysis And Functional Annotation}

Variables with a $p$ value $<0.05$ and a variable importance in projection (VIP) score $>1.0$ were considered differential variables. Metabolic annotation was performed using the LIPID MAPS system (http://www.lipidmaps.org/). Pathway enrichment analysis was carried out using the MetaboAnalyst database (https://www.metaboanalyst.ca/) and the Kyoto Encyclopedia of Genes and Genomes database (https://www. kegg.jp/). The Human Metabolome Database was also used for relevant analyses (http://www.hmdb.ca/).

\section{Statistical Analysis}

Statistical analysis was performed using SPSS 20.0 (Chicago, USA). Comparisons between two groups were performed using student's $t$-test. The statistical significance level was set at a $\mathrm{p}$ value $<0.05$. Data were visualized by Cytoscape 3.7.0 and GraphPad Prism 8.0 (San Diego, California, USA).

\section{Results}

\section{Significant Differences In Metabolic Phenotypes Between Schizophrenia Microbiota And Healthy Microbiota Recipient Mice}

Previously, in an FMT experiment, we found that schizophrenia microbiota recipient mice displayed schizophrenia-relevant behaviors including hyperactivity (greater total distance in the open-field test), increased startle responses (exaggerated startle response to high-decibel tones), decreased anxiety (greater distance traveled in the center region in the open-field test) and depressive-like behaviors (decreased immobility in a forced swimming test). ${ }^{16}$ Tissue samples, including cortex, cerebellum and striatum tissue, derived from our previous study were used. The metabolic signatures of the three brain regions were systematically compared between schizophrenia microbiota and healthy microbiota recipient mice by both 
GC-MS and LC-MS. The typical base peak intensity and original total ion chromatograms are shown in Figures S1 and $\underline{\mathrm{S}}$, respectively. PLS-DA showed clear discrimination between the two groups (Figure 2). $\mathrm{R}^{2} \mathrm{Y}$ and $\mathrm{Q}^{2}$ represent variation of the cumulative model and the cumulative predicted variation, respectively. The $\mathrm{R}^{2} \mathrm{Y}$ and $\mathrm{Q}^{2}$ values indicate a robust metabolic difference between the two groups. Furthermore, the 200-iteration permutation test suggested that the possibility of overfitting was avoided, confirming the reliability of these generated models.
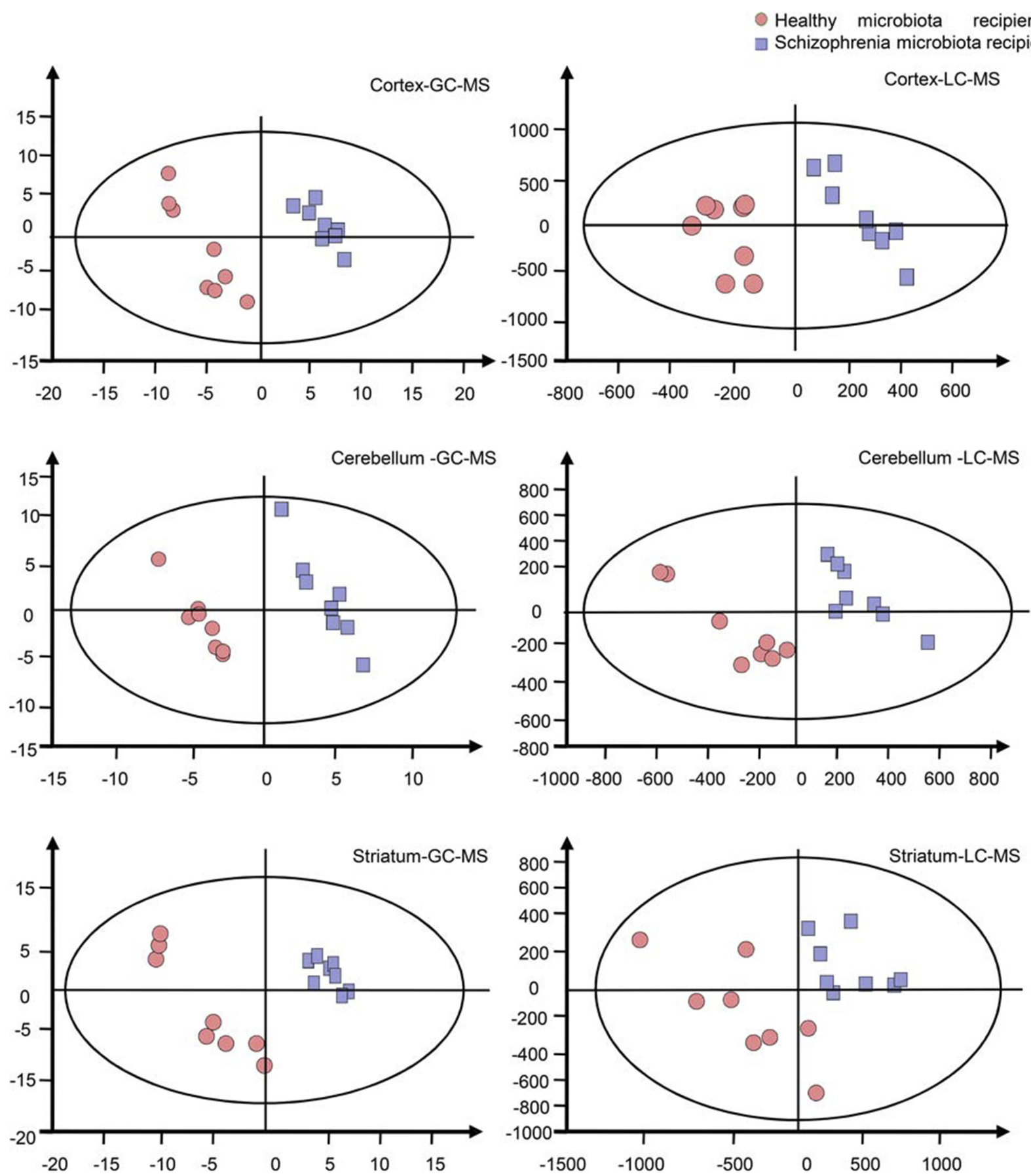

Figure 2 Metabonomic analysis of brain samples. Partial least-squares discriminant analysis score plots showing clear discrimination between schizophrenia microbiota recipient mice (blue boxes) and healthy microbiota recipient mice (red dots) ( $\mathrm{n}=8$ each group) by gas chromatography-mass spectrometry (GC-MS) (cortex: ${ }^{2} Y=0.997$, $Q^{2}=0.894$; cerebellum: $R^{2} Y=0.989, Q^{2}=0.578$; striatum: $R^{2} Y=0.976, Q^{2}=0.881$ ) and liquid chromatography-mass spectrometry (LC-MS) (cortex: $R^{2} Y=0.941, Q^{2}=0.15$; cerebellum: $R^{2} Y=0.953, Q^{2}=0.139$; striatum: $R^{2} Y=0.963, Q^{2}=0.699$ ). 


\section{Classification Of The Differential Metabolites}

Initially, by comparing the levels of metabolites, we identified 213, 255 and 428 significantly altered metabolites ( $p$ value $<0.05)$ in the cortex, cerebellum and striatum, respectively, between the two groups (Figure 3A). Addtionlly, the VIP score $(>1.0)$ was further used to define the differential metabolites. Consequently, we identified a total of 499 differential metabolites between schizophrenia microbiota and healthy microbiota recipient mice in the three brain regions (VIP score $>1.0$ and $p$ value $<0.05$ ). Additionally, 263 of the 499 metabolites were up-regulated, and the remaining metabolites were down-regulated in the schizophrenia microbiota recipient mice relative to the healthy microbiota recipient mice. The detailed data are shown in Table S1. Functionally, the differential metabolites are involved in lipid, amino acid and carbohydrate metabolism (Figure 3B). Interestingly, more than half of the differential metabolites (56.1\%) were lipids. Compared with healthy microbiota recipient mice, most of the metabolites in the three pathways were down-regulated in the cerebellum and striatum but up-regulated in the cortex in schizophrenia microbiota recipient mice (Table S2).

\section{Intersection Metabolic Analysis Of Differential Metabolites}

Intersection metabolic analysis showed that at the class level, the differential metabolites were evenly distributed in the cortex, cerebellum and striatum, suggesting that the gut microbiota broadly influences multiple brain regions in schizophrenia microbiota recipient mice (Figure 4A). In addition, we found that the majority of the differential metabolites were involved in lipid metabolism, especially glycerophospholipid and fatty acyl metabolism (Figure 4B). Compared with healthy microbiota recipient mice, glycerophospholipid and fatty acyl metabolism was mainly down-regulated in the cerebellum and

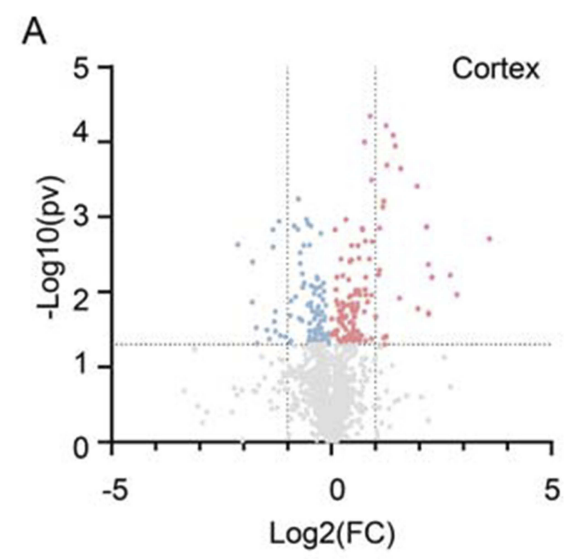

B

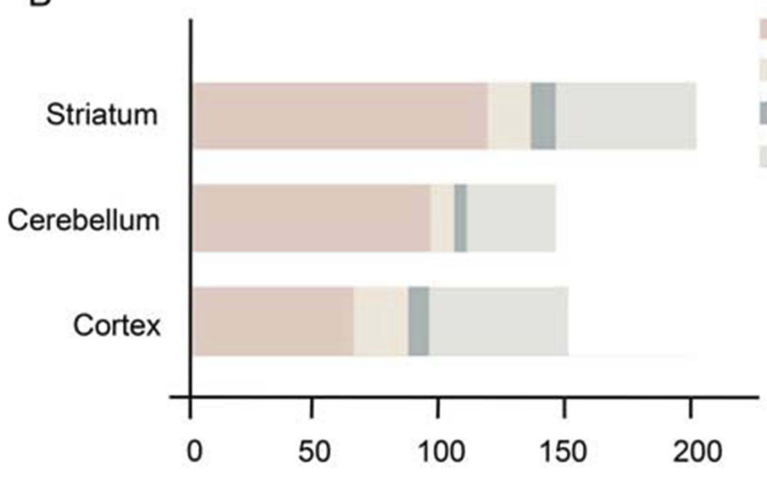

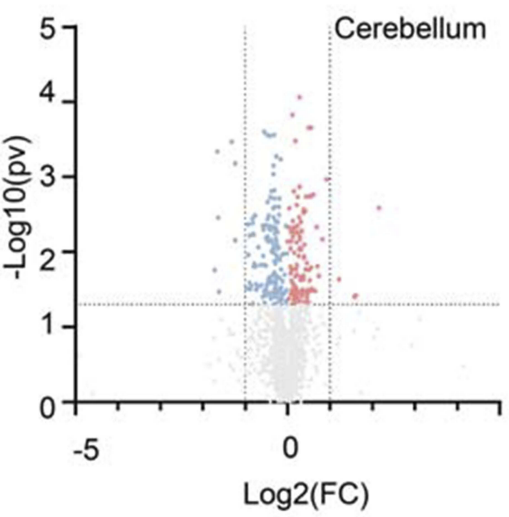

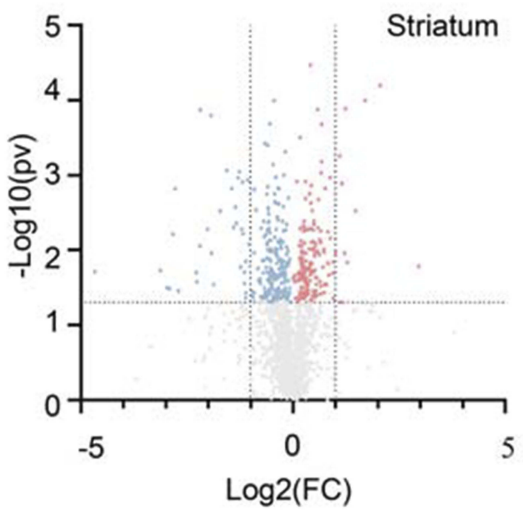
Lipids Amino acids Carbohydrates Others
C

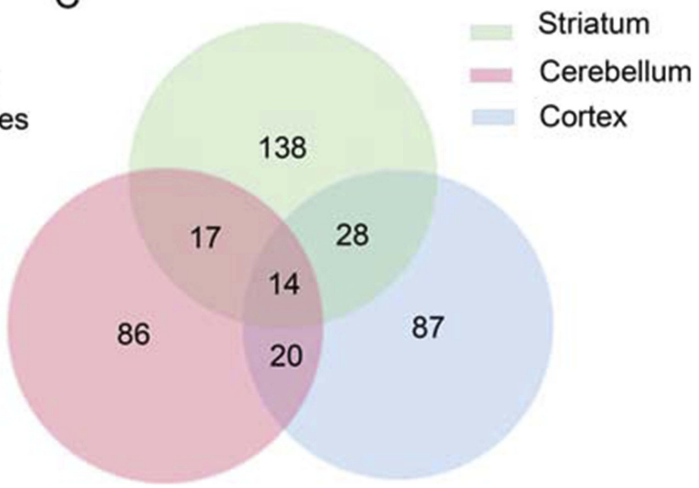

Figure 3 (A) Volcano plot of metabolites. Metabolites were identified by gas chromatography-mass spectrometry and liquid chromatography-mass spectrometry in the three brain regions. The red dots (up-regulation) and blue dots (down-regulation) represent 213, 255 and 428 metabolites selected from 2415 , 2373 and 2416 identified metabolites in the cortex, cerebellum and striatum, respectively ( $\mathrm{p}$ value $<0.05$ ). (B) Number of differential metabolites. Variables with a VIP score $>I$ and $p$ value $<0.05$ were considered differential variables. In total, 499 differential metabolites were identified in the cortex, cerebellum and striatum. (C) Venn diagram of differential metabolites. The numbers of altered metabolites in the three brain regions in schizophrenia microbiota recipient mice are shown. We found that 14 differential metabolites overlapped among the three brain regions. 

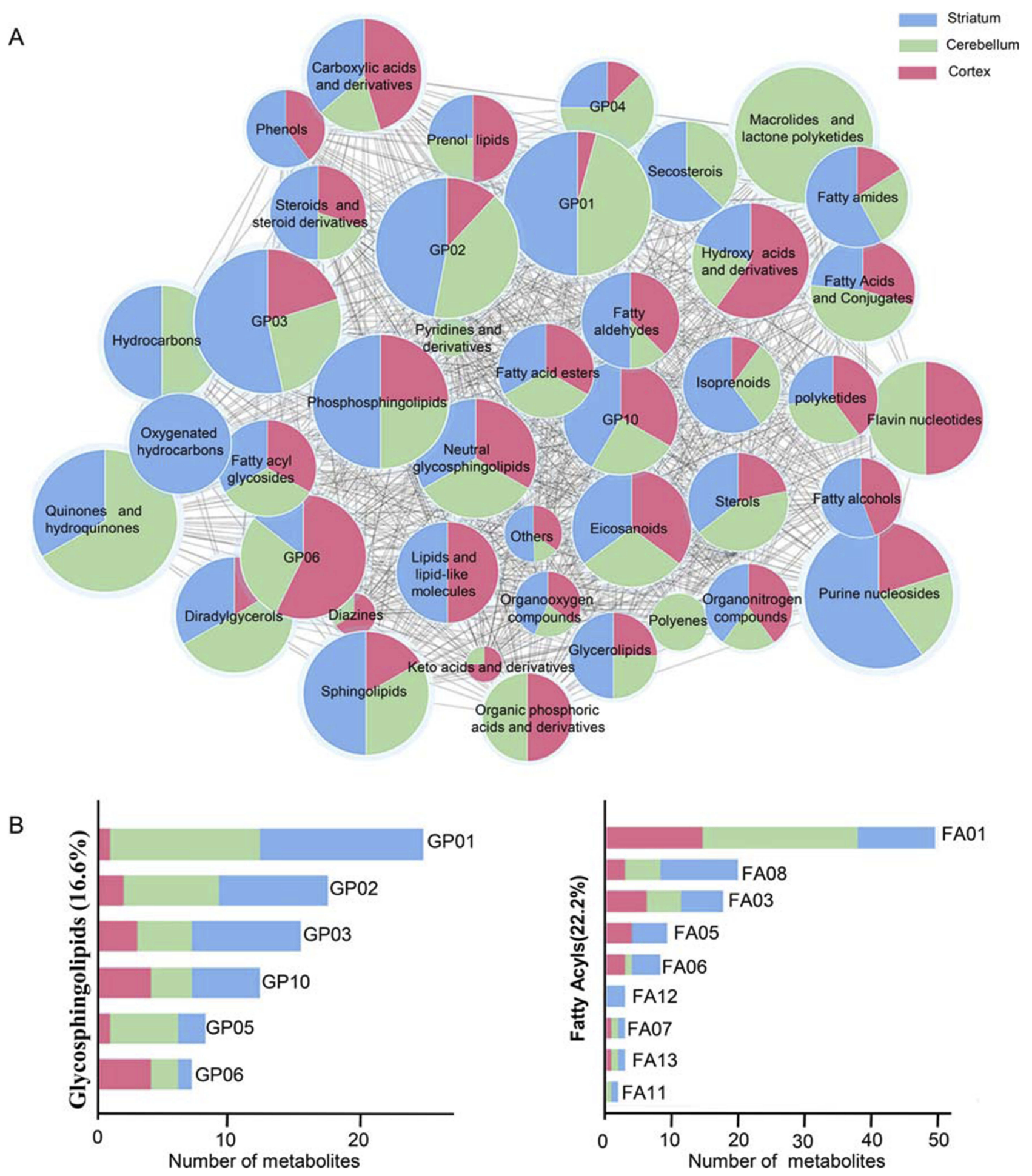

Figure 4 (A) Intersection metabolic analysis of differential metabolites at the class level. Intersection analysis was displayed by a network of nodes and lines using Cytoscape. The majority of metabolites were evenly distributed in the three regions at the class level. The size of the node represents the relative abundance of metabolites, and the lines represent the correlations between metabolites. (B) Proportion of differential metabolites of fatty acyls and glycerophospholipids in the three brain regions. GPOI, Glycerophosphocholines; GP02, Glycerophosphoethanolamines; GPI0, Glycerophosphates; GP04, Glycerophosphoglycerols; GP06, Glycerophosphoinositols; GP03, Glycerophosphoserines; FA0I, Fatty acids and conjugates; FA08, Fatty amides; FA03, Eicosanoids; FA05, Fatty alcohols; FA06, Fatty aldehydes; FAI2, Oxygenated hydrocarbons; FA07, Fatty acid esters; FAI3, Fatty acyl glycosides; FAII, Hydrocarbons.

striatum of schizophrenia microbiota recipient mice, but the changes in these metabolites were diverse in the cortex
(Table S3). Moreover, we found that 14 differential metabolites were consistently altered in the three regions (Figure 3C). Most 
of these metabolites were classified as lipids including fatty acyls, sterol lipids, glycerolipids, prenollipids, polyketides and neutral glycosphingolipids (Table S4). Specifically, four metabolites including "3,7-Dimethyl-8.11-dioxo-2E,6E,9Edodecatrienal", '15-Deoxy-d-12.14-PGJ2', "anandamide (20: 1,n-9)" and "1-(O-alpha-D-glucopyranosyl)-3-keto-(1, 27R)octacosanediol", which are fatty acyls, were consistently altered (Figure 5). Together, these results indicated that disruptions in glycerophospholipid and fatty acyl metabolism are involved in the pathophysiology of schizophrenia.

\section{Pathway Analysis Of Lipid Metabolites}

As the majority of the differential metabolites were lipids, we performed a functional analysis of lipid metabolites in the three brain regions. We observed that these metabolic pathways, including glycerophospholipid, sphingolipid and arachidonic acid metabolism, were significantly altered in the schizophrenia microbiota recipient mice relative to the healthy microbiota recipient mice (Figure 6A). This finding also confirms that disruptions of glycerophospholipid and fatty acyl metabolism are a hallmark of schizophrenia microbiota recipient mice.

\section{Discussion}

In this study, the metabolic signatures of the cortex, cerebellum and striatum were systematically analyzed in schizophrenia microbiota and healthy microbiota recipient mice by two complementary metabolic approaches. We found that the metabolite signatures of these three regions were substaintially different between the two groups. Interestingly, we found that disruptions of glycerophospholipid and fatty acyl metabolism were mainly involved in the onset of schizophrenia-related behaviors. These findings provide a new perspective for understanding of the pathogenesis of schizophrenia (Figure 6B).

We showed that dysbiosis of the gut microbiota could significantly affect the metabolic phenotypes of the cortex, cerebellum and striatum in schizophrenia microbiota
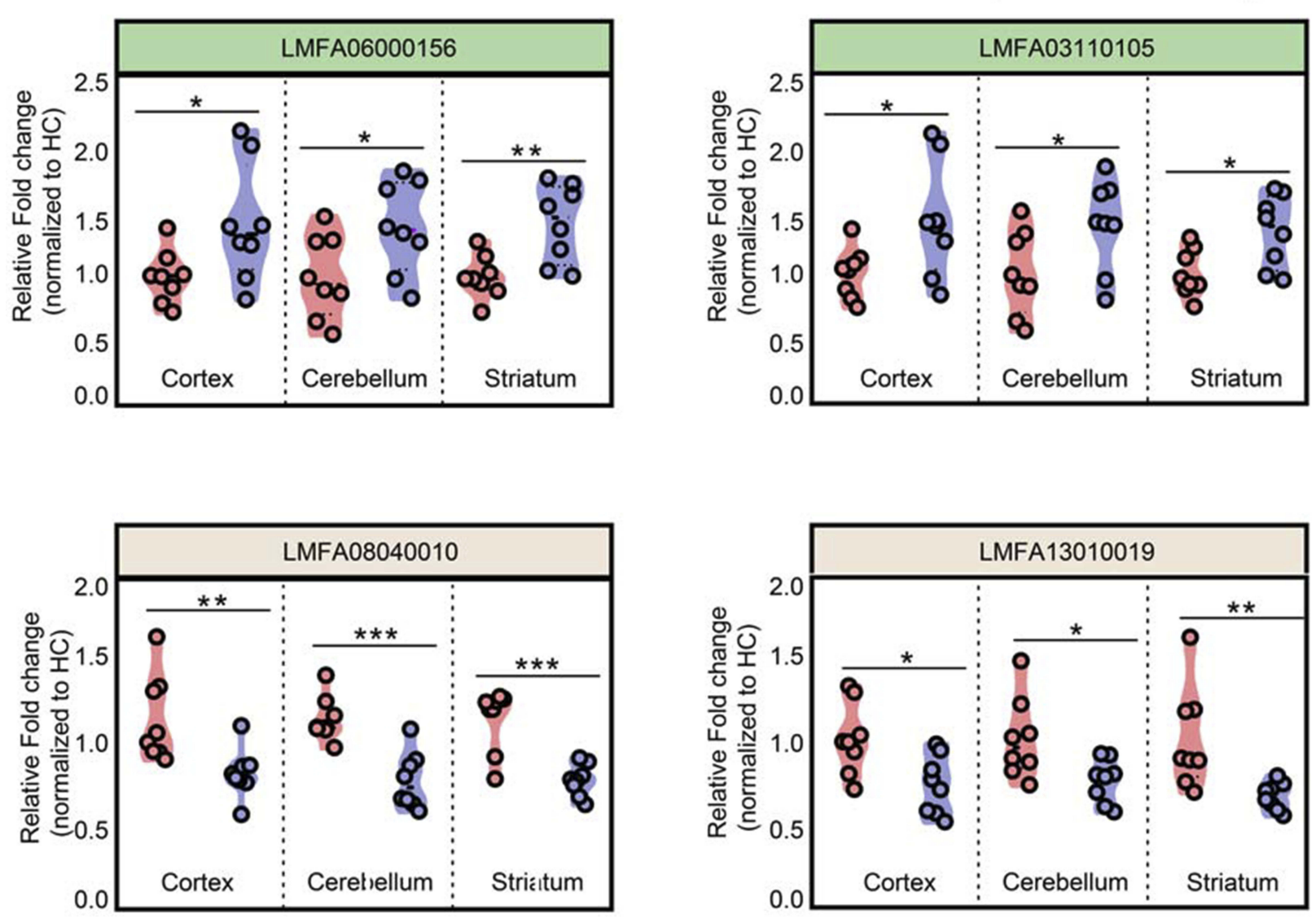

Figure 5 Overlapping differential metabolites in the three brain regions were fatty acyls. Fatty acyl metabolites were consistently altered in the cortex, cerebellum and striatum. ( $\mathrm{n}=8$ subjects per group. *p<0.05; **p<0.0I; ***p<0.00I), LMFA06000I56, "3,7-Dimethyl-8.II-dioxo-2E,6E,9E-dodecatrienal”; LMFA03 II0I05, 'I5-Deoxy-d-I2.I4PGJ2'; LMFA08040010, “anandamide(20:I,n-9)”; LMFAI3010019, “I-(O-alpha-D-glucopyranosyl)-3-keto- (I,27R)-octacosanediol”. 


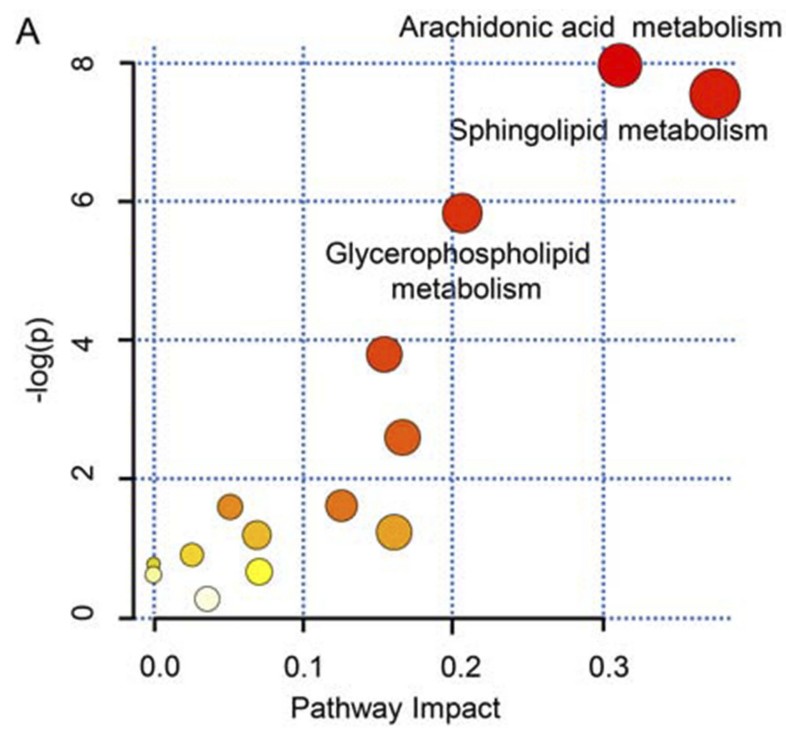

B

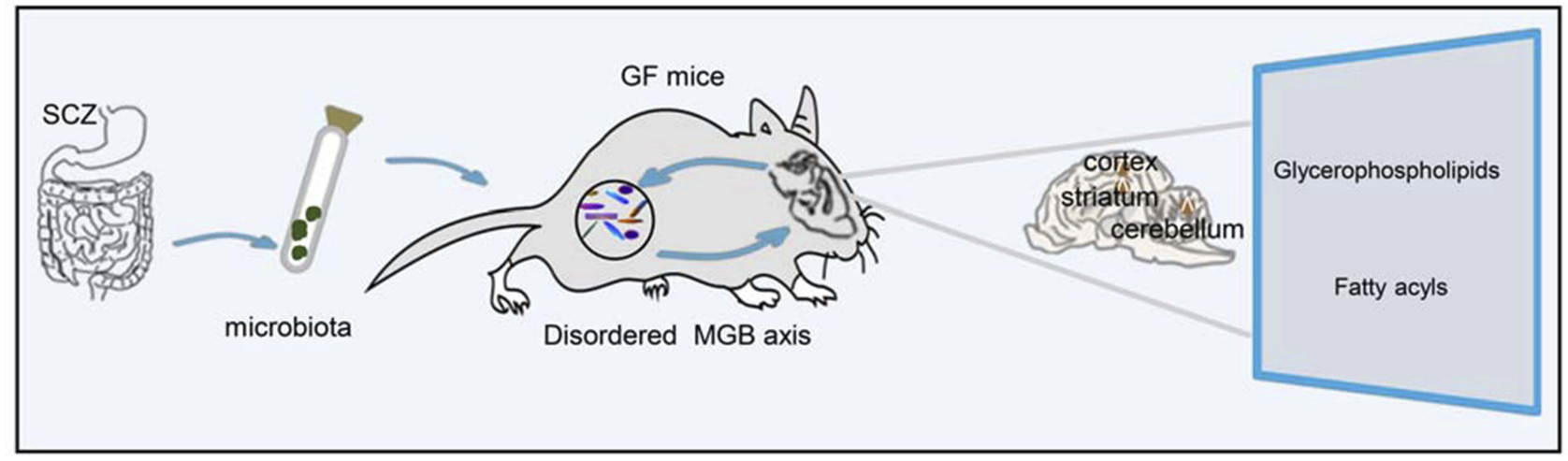

Figure 6 (A) Metabolite pathways of lipids. Color variations (from yellow to red) indicate metabolites from the database with different levels of significance. (B) Schematic representation of the findings. The gut microbiota may be associated with the onset of schizophrenia-relevant behaviors via regulation of glycerophospholipid and fatty acyl metabolism.

recipient mice. Consistent with these findings, previous neuroimaging studies have shown that these regions are highly correlated with the onset of schizophrenia. ${ }^{27-30}$ Additionally, at a functional level, alterations of lipid, amino acid and carbohydrate metabolism were also observed in schizophrenia microbiota recipient mice. ${ }^{16}$ We also observed that the altered metabolites were mainly involved in lipid metabolism, and a majority of the consistently altered metabolites were involved in lipid metabolism in the three brain regions. These findings help to increase the understanding the metabolic mechanisms of schizophrenia.

In the three brain regions, we found that glycerophospholipid metabolism was down-regulated in schizophrenia microbiota recipient mice compared with healthy microbiota recipient mice. Emerging studies have demonstrated that glycerophospholipids are involved in the initiation and elongation of dendritic spines. ${ }^{31,32}$ Our findings suggest that dysbiosis of the gut microbiota may participate in the pathogenesis of schizophrenia by modulating the growth of dendritic spines. Consistent with our hypothesis, previous studies have observed a marked reduction in dendritic spines in human postmortem brain tissue from schizophrenia patients. ${ }^{33,34}$ Further studies are required to investigate how the gut microbiota modulates the genes related to dendritic spine development.

Fatty acyls are the basic building blocks of complex lipids, which play a crucial role in sustaining the physiological function of neurons. ${ }^{35-37} \mathrm{We}$ found that the levels of major fatty acyls were decreased in the cerebellum and striatum but increased in the cortex in schizophrenia microbiota recipient mice. Studies have shown that some fatty acyls can increase synaptic protein levels and the number of c-Fos-positive neurons and thus enhance synaptic plasticity in the hippocampus. ${ }^{38-40}$ Our findings 
indicate that disturbances of gut microbiota may be associated with the onset of schizophrenia-relevant behaviors via regulation of synaptic plasticity. Consistent with our speculation, synaptic plasticity was significantly reduced in the hippocampus of a neurodevelopmental model of schizophrenia. Moreover, treatment of these rats with fatty acid could effectively alleviate the synaptic plasticity deficits. ${ }^{41-43}$ These diverse changes of fatty acyls in different brain regions suggest that fatty acyls may play a complicated role in the onset of schizophrenia-relevant behaviors through modulating the functions of neural circuits, which requires further investigation.

Previously, we found that schizophrenia microbiota recipient mice were characterized by disrupted serum lipid metabolism, including phosphatidylcholine (PC) and phosphatidylethanolamine (PE) ${ }^{16}$ Interestingly, similar findings were also observed in the cortex, cerebellum and striatum. PC and $\mathrm{PE}$ are the most abundant phospholipids in cell organelles. ${ }^{44,45}$ A previous study reported that the PC and PE levels were decreased in platelet membranes of schizophrenia patients. ${ }^{46}$ Moreover, another study observed that PC and PE were significantly altered in the white matter of schizophrenic patients. ${ }^{47}$ These previous findings suggest that the alterations in PC and PE may be a common molecular basis of schizophrenia in the peripheral and central compartments.

Our research has the following limitations: (i) based on animal research, we found that alterations in glycerophospholipid and fatty acyl metabolism were implicated in the onset of schizophrenia-relevant behaviors. However, recent meta-analysis of clinical trials showed that supplementation with probiotics may not be effective in the treatment of schizophrenia or depression. ${ }^{48,49}$ Therefore, further studies aimed at identifying key gut species and their modulation of metabolic pathways are required, which may provide effective treatment targets for schizophrenia; (ii) a non-targeted metabolomics study was used to characterize the altered metabolic signatures of three brain regions in schizophrenia microbiota recipient mice. Further studies using gas chromatography-quadrupole time of flight mass spectrometry should be considered due to its high resolution, sensitivity and selectivity. ${ }^{50}$ Additionally, targeted lipidomics studies should be used to clarify the detailed metabolic changes.

\section{Conclusion}

In this study, using systematic, comparative metabonomic analyses, we observed that dysbiosis of the gut microbiota may participate in the onset of schizophrenia-relevant behaviors by modulating glycerophospholipid and fatty acyl metabolism. Our findings provide novel clues for investigating the pathological mechanisms of schizophrenia.

\section{Acknowledgement}

This work was supported by the National Key R\&D Program of China (2016YFC1307200, 2017YFA0505700, 2017YFD0500503 and 2017YFD0501000), the Natural Science Foundation Project of China (81771490, 81371310, 31271189, 81200899, 81601207 and 31300917), institutional funds from the Chongqing Science and Technology Commission (cstc2014kjrc-qnrc10004).

\section{Disclosure}

Hong Wei and Peng Xie are co-senior authors. The authors report no conflicts of interest in this work.

\section{References}

1. Lysaker PH, Gumley A, Luedtke B, et al. Social cognition and metacognition in schizophrenia: evidence of their independence and linkage with outcomes. Acta Psychiatr Scand. 2013;127(3):239-247. doi:10.1111/acps.2013.127.issue-3

2. Jin H, Mosweu I. The societal cost of schizophrenia: a systematic review. PharmacoEconomics. 2017;35(1):25-42. doi:10.1007/s40273016-0444-6

3. Mumtaz F, Khan MI, Zubair M, Dehpour AR. Neurobiology and consequences of social isolation stress in animal model-A comprehensive review. Biomed Pharmacother. 2018;105:1205-1222. doi:10.1016/j.biopha.2018.05.086

4. Zhuo C, Yao Y, Xu Y, et al. Schizophrenia and gut-flora related epigenetic factors. Prog Neuropsychopharmacol Biol Psychiatry. 2019;90:49-54. doi:10.1016/j.pnpbp.2018.11.005

5. Prata J, Santos SG, Almeida MI, Coelho R, Barbosa MA. Bridging Autism Spectrum Disorders and Schizophrenia through inflammation and biomarkers - pre-clinical and clinical investigations. $J$ Neuroinflammation. 2017;14(1):179.

6. Yang AC, Tsai S-J. New targets for schizophrenia treatment beyond the dopamine hypothesis. Int $J$ Mol Sci. 2017;18(8):1689. doi:10.3390/ijms18081689

7. Whitton L, Cosgrove D, Clarkson C, et al. Cognitive analysis of schizophrenia risk genes that function as epigenetic regulators of gene expression. Am J Med Genet B Neuropsychiatr Genet. 2016;171(8):1170-1179. doi:10.1002/ajmg.b.32503

8. Xu L, Qi X, Zhu C, Wan L. Activation of IL-8 and its participation in cancer in schizophrenia patients: new evidence for the autoimmune hypothesis of schizophrenia. Neuropsychiatr Dis Treat. 2018;14:3393-3403. doi:10.2147/NDT.S188210

9. Rosa CP, Brancaglion GA, Miyauchi-Tavares TM, Corsetti PP, de Almeida LA. Antibiotic-induced dysbiosis effects on the murine gastrointestinal tract and their systemic repercussions. Life Sci. 2018;207:480-491. doi:10.1016/j.1fs.2018.06.030

10. Lozupone CA, Stombaugh JI, Gordon JI, Jansson JK, Knight R. Diversity, stability and resilience of the human gut microbiota. Nature. 2012;489(7415):220-230. doi:10.1038/nature11550

11. Mayer EA, Tillisch K, Gupta A. Gut/brain axis and the microbiota. $J$ Clin Invest. 2015;125(3):926-938. doi:10.1172/JCI76304

12. Pan JX, Deng FL, Zeng BH, et al. Absence of gut microbiota during early life affects anxiolytic behaviors and monoamine neurotransmitters system in the hippocampal of mice. J Neurol Sci. 2019;400:160-168. doi:10.1016/j.jns.2019.03.027 
13. Zheng P, Zeng B, Zhou C, et al. Gut microbiome remodeling induces depressive-like behaviors through a pathway mediated by the host's metabolism. Mol Psychiatry. 2016;21(6):786-796. doi:10.1038/ mp.2016.44

14. Sherwin E, Sandhu KV, Dinan TG, Cryan JF. May the force be with you: the light and dark sides of the microbiota-gut-brain axis in neuropsychiatry. CNS Drugs. 2016;30(11):1019-1041. doi:10.1007/ s40263-016-0370-3

15. Hemmings G. Schizophrenia. Lancet. 2004;364(9442):1312-1313. doi:10.1016/S0140-6736(04)17181-X

16. Zheng $\mathrm{P}$, Zeng B, Liu M, et al. The gut microbiome from patients with schizophrenia modulates the glutamate-glutamine-GABA cycle and schizophrenia-relevant behaviors in mice. Sci Adv. 2019;5(2): eaau8317. doi:10.1126/sciadv.aau8317

17. Sigurdsson T. Neural circuit dysfunction in schizophrenia: insights from animal models. Neuroscience. 2016;321:42-65. doi:10.1016/j. neuroscience.2015.06.059

18. Cao H, Dixson L, Meyer-Lindenberg A, Tost H. Functional connectivity measures as schizophrenia intermediate phenotypes: advances, limitations, and future directions. Curr Opin Neurobiol. 2016;36:714. doi:10.1016/j.conb.2015.07.008

19. Lett TA, Voineskos AN, Kennedy JL, Levine B, Daskalakis ZJ. Treating working memory deficits in schizophrenia: a review of the neurobiology. Biol Psychiatry. 2014;75(5):361-370. doi:10.1016/j. biopsych.2013.07.026

20. Viana J, Hannon E, Dempster E, et al. Schizophrenia-associated methylomic variation: molecular signatures of disease and polygenic risk burden across multiple brain regions. Hum Mol Genet. 2017;26 (1):210-225. doi:10.1093/hmg/ddw373

21. Errico F, Napolitano F, Squillace M, et al. Decreased levels of D-aspartate and NMDA in the prefrontal cortex and striatum of patients with schizophrenia. J Psychiatr Res. 2013;47(10):14321437. doi:10.1016/j.jpsychires.2013.06.013

22. Schmahmann JD. The role of the cerebellum in cognition and emotion: personal reflections since 1982 on the dysmetria of thought hypothesis, and its historical evolution from theory to therapy. Neuropsychol Rev. 2010;20(3):236-260. doi:10.1007/s11065-010-9142-x

23. Sathyanesan A, Zhou J, Scafidi J, Heck DH, Sillitoe RV, Gallo V. Emerging connections between cerebellar development, behaviour and complex brain disorders. Nat Rev Neurosci. 2019;20(5):298313. doi:10.1038/s41583-019-0152-2

24. Chung US, Han DH, Shin YJ, Renshaw PF. A prosocial online game for social cognition training in adolescents with high-functioning autism: an fMRI study. Neuropsychiatr Dis Treat. 2016;12:651-660. doi:10.2147/NDT.S94669

25. Lei Y, Li D, Deng J, et al. Metabolomic profiling of three brain regions from a postnatal infected Borna disease virus $\mathrm{Hu}-\mathrm{H} 1$ rat model. Metabolomics. 2013;10(3):484-495. doi:10.1007/s11306-013-0593-y

26. Zheng P, Wei Y-D, Yao G-E, et al. Novel urinary biomarkers for diagnosing bipolar disorder. Metabolomics. 2013;9(4):800-808. doi:10.1007/s11306-013-0508-y

27. Sui J, Pearlson GD, Du Y, et al. In search of multimodal neuroimaging biomarkers of cognitive deficits in schizophrenia. Biol Psychiatry. 2015;78(11):794-804. doi:10.1016/j.biopsych.2015.02.017

28. Carass A, Cuzzocreo JL, Han S, et al. Comparing fully automated stateof-the-art cerebellum parcellation from magnetic resonance images. NeuroImage. 2018;183:150-172. doi:10.1016/j.neuroimage.2018.08.003

29. McCutcheon RA, Abi-Dargham A, Howes OD. Schizophrenia, dopamine and the striatum: from biology to symptoms. Trends Neurosci. 2019;42(3):205-220. doi:10.1016/j.tins.2018.12.004

30. Zhuo K, Tang Y, Song Z, et al. Repetitive transcranial magnetic stimulation as an adjunctive treatment for negative symptoms and cognitive impairment in patients with schizophrenia: a randomized, double-blind, sham-controlled trial. Neuropsychiatr Dis Treat. 2019;15:1141-1150. doi:10.2147/NDT.S196086
31. Ziegler AB, Tavosanis G. Glycerophospholipids - emerging players in neuronal dendrite branching and outgrowth. Dev Biol. 2018.

32. Meltzer S, Bagley JA, Perez GL, et al. Phospholipid homeostasis regulates dendrite morphogenesis in drosophila sensory neurons. Cell Rep. 2017;21(4):859-866. doi:10.1016/j.celrep.2017.09. 089

33. Konopaske GT, Lange N, Coyle JT, Benes FM. Prefrontal cortical dendritic spine pathology in schizophrenia and bipolar disorder. JAMA Psychiatry. 2014;71(12):1323-1331. doi:10.1001/jamapsychiatry.2014. 1582

34. Hill JJ, Hashimoto T, Lewis DA. Molecular mechanisms contributing to dendritic spine alterations in the prefrontal cortex of subjects with schizophrenia. Mol Psychiatry. 2006;11(6):557-566. doi:10.1038/sj. mp.4001792

35. Fahy E, Subramaniam S, Brown HA, et al. A comprehensive classification system for lipids. J Lipid Res. 2005;46(5):839-861. doi:10.1194/jlr.E400004-JLR200

36. Xicoy H, Wieringa B, Martens GJM. The role of lipids in Parkinson's disease. Cells. 2019;8(1):27. doi:10.3390/cells8010027

37. Huang Y, Huang S, Lam SM, Liu Z, Shui G, Zhang YQ. Acsl, the Drosophila ortholog of intellectual-disability-related ACSL4, inhibits synaptic growth by altered lipids. J Cell Sci. 2016;129(21):40344045. doi: $10.1242 /$ jcs. 195032

38. Su HM. Mechanisms of n-3 fatty acid-mediated development and maintenance of learning memory performance. J Nutr Biochem. 2010;21(5):364-373. doi:10.1016/j.jnutbio.2009.11.003

39. Yamada D, Wada K, Sekiguchi M. Modulation of long-term potentiation of cortico-amygdala synaptic responses and auditory fear memory by dietary polyunsaturated fatty acid. Front Behav Neurosci. 2016;10:164. doi:10.3389/fnbeh.2016.00164

40. Saumweber T, Weyhersmuller A, Hallermann S, et al. Behavioral and synaptic plasticity are impaired upon lack of the synaptic protein SAP47. J Neurosci. 2011;31(9):3508-3518. doi:10.1523/JNEUROSCI. 2646-10.2011

41. Sanderson TM, Cotel MC, O’Neill MJ, Tricklebank MD, Collingridge GL, Sher E. Alterations in hippocampal excitability, synaptic transmission and synaptic plasticity in a neurodevelopmental model of schizophrenia. Neuropharmacology. 2012;62(3):13491358. doi:10.1016/j.neuropharm.2011.08.005

42. Patten AR, Sickmann HM, Dyer RA, Innis SM, Christie BR. Omega3 fatty acids can reverse the long-term deficits in hippocampal synaptic plasticity caused by prenatal ethanol exposure. Neurosci Lett. 2013;551:7-11. doi:10.1016/j.neulet.2013.05.051

43. Kobayashi K, Takagi T, Ishii S, Suzuki H, Miyakawa T. Attenuated bidirectional short-term synaptic plasticity in the dentate gyrus of Schnurri-2 knockout mice, a model of schizophrenia. Mol Brain. 2018;11(1):56. doi:10.1186/s13041-018-0400-9

44. Khot V, Chavan-Gautam P, Joshi S. Proposing interactions between maternal phospholipids and the one carbon cycle: a novel mechanism influencing the risk for cardiovascular diseases in the offspring in later life. Life Sci. 2015;129:16-21. doi:10.1 016/j.1fs.2014.09.026

45. Rangel-Huerta OD, Pastor-Villaescusa B, Gil A. Are we close to defining a metabolomic signature of human obesity? A systematic review of metabolomics studies. Metabolomics. 2019;15(6):93. doi:10.1007/s11306-019-1553-y

46. Schmitt A, Maras A, Petroianu G, Braus DF, Scheuer L, Gattaz WF. Effects of antipsychotic treatment on membrane phospholipid metabolism in schizophrenia. J Neural Transm. 2001;108(8-9):10811091. doi:10.1007/s007020170026

47. Ghosh S, Dyer RA, Beasley CL. Evidence for altered cell membrane lipid composition in postmortem prefrontal white matter in bipolar disorder and schizophrenia. J Psychiatr Res. 2017;95:135-142. doi:10.1016/j.jpsychires.2017.08.009 
48. Ng QX, Soh AYS, Venkatanarayanan N, Ho CYX, Lim DY, Yeo WS. A systematic review of the effect of probiotic supplementation on schizophrenia symptoms. Neuropsychobiology. 2019;78(1):1-6. doi:10.1159/ 000498862

49. Ng QX, Peters C, Ho CYX, Lim DY, Yeo WS. A meta-analysis of the use of probiotics to alleviate depressive symptoms. $J$ Affect Disord. 2018;228:13-19. doi:10.1016/j.jad.2017.11.063
50. Zhang B, Zhang $\mathrm{H}$, Du C, et al. Metabolic responses of the growing Daphnia similis to chronic AgNPs exposure as revealed by GC-QTOF/MS and LC-Q-TOF/MS. Water Res. 2017;114:135-143. doi:10.1016/j.watres.2017.02.046

\section{Publish your work in this journal}

Neuropsychiatric Disease and Treatment is an international, peerreviewed journal of clinical therapeutics and pharmacology focusing on concise rapid reporting of clinical or pre-clinical studies on a range of neuropsychiatric and neurological disorders. This journal is indexed on PubMed Central, the 'PsycINFO' database and CAS, and is the official journal of The International Neuropsychiatric Association (INA). The manuscript management system is completely online and includes a very quick and fair peer-review system, which is all easy to use. Visit http://www.dovepress.com/testimonials.php to read real quotes from published authors. 